\title{
GDP data revisions and forward-looking monetary policy in Switzerland
}

\author{
Peter Kugler*, Thomas J. Jordan**, Carlos Lenz***, Marcel R. Savioz****
}

*WWZ University of Basel and Swiss National Bank, Petersgraben 51, Basel 4003, Switzerland, Corresponding author: Tel:. +41-61-267-3344; Fax: +41-61-267-3340

**Swiss National Bank and University of Bern, Börsenstrasse 15, Zürich 8022, Switzerland

*** WWZ University of Basel, Petersgraben 51, Basel 4003, Switzerland

**** Swiss National Bank and University of St. Gallen, Börsenstrasse 15, Zürich 8022,

Switzerland

E-mail Addresses: peter.kugler@unibas.ch (P. Kugler), thomas.jordan@snb.ch (T. Jordan),

carlos.lenz@unibas.ch (C. Lenz), marcel.savioz@snb.ch (M. Savioz)

\begin{abstract}
This paper analyzes forward-looking rules for Swiss monetary policy in a small structural VAR model consisting of four variables taking into account data revisions for GDP. First, the paper develops an analytical method to analyze the effect of data revision errors in GDP on the ex ante or conditional inflation-output-growth volatility trade-off and applies it to Swiss data. Second, the effects of different targets in a forward-looking monetary policy on ex post or unconditional volatility of inflation and output growth is explored by a simulation exercise. In general, the results obtained suggest that focusing monetary policy on GDP growth instead on inflation may lead to an inefficient policy with both increased medium term inflation and GDP growth volatility in the presence of GDP data revisions.
\end{abstract}

Keywords: Structural VAR, forward-looking monetary policy, efficiency frontier, GDP data revisions JEL classification: E52; E58

First Draft May 2002, Last Revision January 2005

Earlier versions of this paper were presented at the annual congress of the "Schweizerische Gesellschaft für Volkswirtschaft und Statistik," Neuchâtel, June 1, 2002, the meeting of the "Ausschuss für Makroökonomie Verein für Socialpolitik", Bonn June 28/29 2002, the SNB-Fed Cleveland Workshop on Monetary Economics, Zürich, July 2/3, 2002, the meeting of the "Ausschuss für Geldtheorie und -politik - Verein für Socialpolitik", Frankfurt am Main, February 20/21, 2003, at the ECB Invited Speakers Seminar, April 52004 and at the Bundesbank Conference "Real-time data and monetary policy", Eltville, May 28/29, 2004. Helpful comments of participants of these meetings and of an anonymous referee are gratefully acknowledged. We are in particular indebted to Ernst Baltensperger, Lawrence Christiano, Manfred Neumann, Günter Coenen and Robert Rasche for their suggestions. 


\section{Introduction}

After 25 years of monetary targeting, the Swiss National Bank (SNB) adopted a new monetary policy framework at the end of 1999. Severe shocks to the demand for central bank money, especially for large denominated bank notes and for reserves held by commercial banks at the SNB, rendered it impossible to use the medium-term target path for the seasonally adjusted monetary base as a guideline for monetary decisions. Thus, the SNB decided to abandon monetary targeting. The new framework consists of three elements. The first element is an explicit definition of price stability. The SNB regards price stability as achieved if CPI inflation is below 2 percent. The second element consists of the use of an inflation forecast as the main indicator to guide monetary policy decisions. The third element is a target range for the three-month Swiss franc Libor as an operational target to implement monetary policy. As in the old concept, maintaining price stability over the medium term remains the main objective of monetary policy also in the new framework.

In the new framework, the inflation forecast serves as the main indicator for guiding policy decisions. Although there is no mechanical reaction to the inflation forecast and the inflation forecast is not treated as an intermediate target, the discussion at the board about monetary policy is focused on the inflation forecast. The forecast used in the decision-making process is a consensus forecast that is derived from a series of models and indicators. The SNB recently started to publish studies regarding these models. Jordan and Peytrignet (2001) delivered an introduction to the inflation forecast of the SNB and the models used to derive it. Stalder (2001) presented the large traditional structural macro model of the SNB and Jordan, Kugler, Lenz and Savioz (2002) provided an overview over the different VAR approaches used at the SNB.

For the purpose of analyzing forward-looking policies, a small structural VAR consisting of four variables was developed by Kugler and Jordan (2004) and Kugler and Rich (2002). This research was extended by Kugler, Jordan, Lenz and Savioz (2005) who developed an analytical method to analyze the ex ante or conditional medium-term inflationoutput-growth volatility trade-off for a forward-looking policy aiming at a convex combination of a medium term inflation and an output growth target in a SVAR model. This paper extends this framework with two respects. First, it considers the effects of data revisions errors in GDP on the ex ante or conditional medium-term inflation-output-growth volatility trade-off. Second, the effects of different targets in a forward-looking monetary policy on ex 
post or unconditional volatility of inflation and output growth is explored by a simulation exercise.

The brief outline of the aims of the paper at hand clearly indicates that it is related to a growing literature on the effects of uncertainty about potential output and the output gap on the performance of monetary policy rules. Orphanides $(2000,2001)$ was one of the first who considered this question using real time data for US output gap. He concluded that data revision errors in the output gap were of crucial importance for the over-expansionary US monetary policy in the sixties and seventies and that neglecting such data revision errors leads in general to a too activist policy. However, Svensson and Woodford (2000) argue that this result is mainly caused by the fact that in Orphanides' framework the central bank behaves as if there were no data revision errors and that it disappears when the optimal policy rule is a function of the best estimate of the state variables, in particular of the output gap. Nevertheless the presence of uncertainty with respect to potential output results, of course, in welfare losses and has effects on simple Taylor-like rules in standard macroeconomic models (Smets, 2002). Extensions and quantitative illustrations of these results are found in Ehrmann and Smets (2003) who build a small stochastic general equilibrium model with endogenous persistence calibrated to the euro area. Briefly their exercise indicates that the data revision problem leads to substantial welfare losses mainly in the form of high output gap variability. Moreover, simple Taylor rules appear to work well when an optimal output gap estimate is used and potential output uncertainty favors the appointment of a conservative (in the sense of Rogoff (1985)) central banker.

This paper differs from the literature sketched above in the following way. First, we do not consider uncertainty with respect to the output gap or potential output but only with respect to GDP, which is by itself already large as result of strong revisions of the quarterly national accounts. Note also that we do not face the problem of obtaining a best estimate of an unobservable variable such as the output gap since only GDP growth is considered in our model. This approach can be seen as a way to circumvent the problem of measuring the level of potential output as recommended among others by Orphanides (2000). Second, we do not consider an explicit structural model of the economy, but our analysis is based on the impulse response we estimated using a SVAR model. Third, the policy makers do not account for the uncertainty with respect to the real time GDP figures. Thus we proceed on the assumption that the first release quarterly GDP figure is the best estimated available at the time when the policy decision is taken. 
The remainder of the paper is organized as follows. Section 2 briefly describes the SVAR model for the analysis of Swiss monetary policy and gives a short account how the inflation-growth trade-off is determined in the absence of GDP data revision errors. Data revision errors are taken into account in Section 3. Section 4 considers the effects of nonequilibrium initial conditions and policy dynamics and Section 5 concludes.

\section{A SVAR Analysis of Swiss Monetary Policy}

In this section, we give a brief account of the framework used for policy analysis in order to obtain the inflation GDP growth volatility trade off. The VAR model includes a vector of changes $X_{t}{ }^{\prime}=\left(\Delta \log y_{t}, \Delta r_{t}, \Delta \log \left(m_{t} / p_{t}\right), \Delta \log p_{t}\right)$ where $y$ is GDP in 1990 Swiss francs, $p$ denotes the consumer price index, $m$ the money stock M1 and $r$ the quarterly average of the three-month Swiss-franc Libor rate of interest. In order to keep the model as lean as possible, the exchange rate is excluded from the vector $X$, as the transmission of monetary policy via the exchange rate is indirectly captured by the impulse responses of the VAR model. Explicit inclusion of the exchange rate would be necessary if this variable had influenced SNB behavior in a systematic way and, therefore, were required to identify a monetary policy shock. Exchange rate considerations played no important role for the bulk of the sample period, the exception being in particular the years 1978/79. Note that we do not select a monetary aggregate with a stable long-run money demand function in levels such as M3. We are only interested in a money stock concept providing a lot of information for the identification of a monetary policy shock. The monetary base was not used as the introduction of the electronic Swiss Interbank Clearing System and the relaxation of banks' liquidity requirements strongly distorted even the rates of change in this aggregate. However, we should also mention that the results are robust with respect to the in- or exclusion of the money stock series: a three variables VAR without money produces essentially the same shape of the impulse responses to the monetary policy shock as the four variable system. Finally, we ought to mention that the standard unit-root and co-integration tests support the first-difference specification adopted in this paper. ${ }^{1}$

\footnotetext{
${ }^{1}$ The results with respect to the interest rate are ambiguous: we cannot reject the unit root hypothesis (ADF test) as well as the stationarity hypothesis (KPSS test). Thus, we proceed on the I(1) hypothesis which is more convenient in our framework for identification of a monetary policy shock. Of course, this implies that the real rate of interest rate is non-stationary what is clearly doubtful in the long-run. However, for the medium-term forecasting horizon considered in this paper this assumption is deemed acceptable.
} 
In this paper, long-run neutrality restrictions are used in order to identify structural shocks with variances normalized to 1 . Briefly, we have the following interpretation of the four identified shocks. First, there is a supply shock which can either have a long-run effect on all four variables considered. Second, we have an IS shock which may have a permanent effect on all variables except for output. Third, there is a money demand shock which affects only the real money stock and prices in the long run. Fourth and most importantly in our framework, we identify a money supply or monetary policy shock which has only a long-run effect on prices (and, of course on the nominal money stock). These six long-run triangularity restrictions lead to an exactly identified model. ${ }^{2}$

All variables included in the model are seasonally adjusted with the exception of the interest rate. The lag length $k$ was set to five quarters, which is the optimal value according to the Akaike criterion. Figure 1 shows the estimates for the cumulated impulse responses of the four variables to all four shocks. ${ }^{3}$ In the first column we find the responses of all variables (in the order they appear in the vector $X$ ) to the supply shock. Then, we have the responses to the IS-shock, to the money demand shock, and finally in the last column the response to the monetary policy shock which is of most importance in the current context. By and large, the latter response estimates correspond to the views shared by most macroeconomists in Switzerland about the effects of monetary policy. First, there is evidence of a short-run negative liquidity effect on the interest rate extending over two quarters. The positive reaction in real GDP starts weakly and reaches its peak after five quarters and starts to peter out after another year. With respect to prices, it takes six quarters until a major positive effect is felt and 14 quarters are needed for full adjustment of prices. After about the fourth quarter, rising prices and inflation expectations cause the interest rate to overshoot temporarily its long-run equilibrium level. Finally, the real money stock remains constant over the long-run equilibrium level for a year and decreases to it over the next four quarters. Before turning to the analysis of monetary policy in this SVAR model, let us briefly mention that the impulse responses to the other three shocks are in line with our priors from economic theory. In particular, we find a permanent positive (negative) effect of the supply shock on the production (prices) and the IS shock leads to a hump-shaped transitory response of production extending over 10 quarters which is accompanied by a permanent increase in the price level.

\footnotetext{
${ }^{2}$ In earlier papers mentioned in the introduction we used an over-identified SVAR model including short-run restrictions. The over-identifying restrictions do not change essentially the results obtained in this paper since that SVAR model produces similar impulse responses for the monetary shock. However, the other shocks are easier to interpret in the current version of the model.
} 
Alternative strategies for Swiss monetary policy can now be analyzed by deriving conditional forecasts from the SVAR model. Specifically, we determine a sequence of policy shocks required to satisfy such conditions as an average inflation target over a two- or threeyear period. Before we turn to this exercise in detail, we have to discuss briefly the appropriateness of our approach. It might be argued that the change in the SNB's monetary regime, as outlined above, invalidates the use of a model fitted to data generated by a different monetary environment. However, we believe that this problem is not of paramount importance in the present context. Price stability remained the ultimate objective of Swiss monetary policy throughout the sample period. Moreover, although the SNB adjusted its operating procedures at the end of 1999, this modification did not cause a break in the time series process of the variables considered in our SVAR model: Bank reserves, used as the main policy instrument before 1999, and the interest rate on repos, the principal new instrument, are not included in our VAR system.

Now let us briefly outline the approach developed by Kugler, Jordan, Lenz and Savioz (2005) for the analysis of forward looking monetary policy. Consider a monetary policy strategy based on an average inflation forecast for the next $K$ quarters as it is applied in Switzerland. Take the example of a monetary policy reacting symmetrically to positive and negative deviations from the inflation target $\pi^{*}$ measured at a quarterly rate. In this framework policy is directed by the expected deviation, as of time $t$, of the average inflation from its target for horizon $K$

$$
d_{p}(K, t)=K \pi^{*}-\left(E_{t} \log p_{t+K}-\log p_{t}\right)
$$

where $E_{t}\left(x_{i, t+K}\right)=E_{T}\left(x_{i, t+K} \mid x_{t}, x_{t-1} \ldots\right)$.

Next, we have to determine the sequence of monetary shocks from $t+1$ to $t+K$ that leads to an expected average inflation which is equal to the target $\pi^{*}$. In Switzerland monetary policy decisions have to be made given last period values for CPI inflation and money growth but only a first estimate of GDP growth for the last few quarters. This situation thus differs from the usual assumption in theoretical models that monetary policy can react to current period final values of inflation and output. There is an infinite number of ways to calculate these shocks. Leeper and Zha (1999) show that policy shocks in VAR-models have to be modest and least disturbing in order not to violate the validity of the simulations. We

\footnotetext{
${ }^{3}$ No confidence intervals are given in Figure 1. Jordan, Kugler, Lenz and Savioz (2002) show that the effects of
} 
therefore minimize the sum of the squared shocks subject to the restriction so that the average inflation rate is on target. As shown by Jordan, Kugler, Lenz and Savioz (2005) this leads to the following sequence of policy shocks

$$
u_{4 t+i}=\frac{A A_{44}(K-i)}{\sum_{j=0}^{K-1} A A_{44}^{2}(j)} d_{p}(K, t)=g_{p i} d_{p}(K, t)
$$

$A A(j)$ is the $4 \times 4$ matrix of the impulse response, cumulated over $j$ periods. Thus, the element 4,4 of this matrix gives the $j$ period cumulated response of inflation to a monetary shock In the remainder of the paper, we call a rule within our SVAR approach based exclusively on an inflation target a strategy of strict medium-term inflation targeting.

Of course, we can apply the same approach using the average output growth as a target of monetary policy. Assume that the targeted output growth rate is denoted by $\gamma^{*}$. Again we define first the deviation of the unconditional forecast of the output growth from target $K$ periods ahead:

$$
d_{y}(K, t)=K \gamma^{*}-\left(E_{t} \log y_{t+K}-\log y_{t}\right)
$$

The application of the same procedure as applied for inflation provides us with the following optimal (in the least squares sense) policy shocks for $t+1$ to $t+K$ :

$$
u_{4 t+i}=\frac{A A_{14}(K-i)}{\sum_{j=0}^{K-1} A A_{14}^{2}(j)} d_{y}(K, t)=g_{y i} d_{y}(K, t)
$$

In what follows, we call a rule within our SVAR approach based exclusively output growth target a strategy of strict medium-term output growth targeting.

Now let us consider the trade-off faced by monetary policy in the framework of our SVAR model. To this end, we consider the variability of inflation and output growth implied by different degrees of medium-term inflation and output growth targeting over the $K$-period horizon. To start with, we define a convex combination of the monetary policy shocks for strict medium-term inflation or output growth targeting given in equations (2) and (4): 


$$
u_{4 t+i}=\alpha g_{p i} d_{p}(K, t)+(1-\alpha) g_{y i} d_{y}(K, t), \quad i=1, \ldots, K
$$

This is the situation of a monetary policy board, where the decision is taken by consensus and according to the average preferences of its members. The board members have either the preference for pure inflation targeting or pure output growth targeting in the medium term. The parameter $\alpha$ thus reflects the fraction of the inflation hawks in the board and $1-\alpha$ is the fraction of the inflation doves. Of course for $\alpha=1$ we have the case of strict medium-term inflation targeting and for $\alpha=0$ we follow a strict medium-term output growth targeting. This strategy corresponds to the goal of minimizing the weighted sum of the conditional variability of the expected medium term inflation and growth rate.

The proposed formalization of the monetary policy strategy is appropriate in the sense that it captures very well the focus of medium term orientation of Swiss monetary policy on inflation and GDP growth perspectives. Indeed, our experience tells us that the discussion about Swiss monetary policy exactly focuses on medium term inflation and GDP growth outlook and a main issue is how much a GDP growth target should be taken into account. However, our framework for monetary policy analysis differs from the two approaches usually adopted in the literature, namely the Taylor rule and the optimizing framework. It may be argued that our framework lies somehow between these two approaches. Equation (5) shares some feature with the Taylor rule framework: it implies, for instance, a negative value for the monetary policy shock when the medium term inflation forecast and/or GDP growth forecast is above target which leads to an increase in the interest rate. The weights $\alpha$ and 1- $\alpha$ play a similar role as the coefficients of the inflation and output gap term in a Taylor rule, respectively. By contrast to an ad hoc Taylor rule, the policy reaction in our framework aims at a medium-term target taking fully into account the expected dynamic effects according to a fully specified empirical model. This feature of our model is shared with the optimizing framework. However, the latter is more general in the sense that a discounted weighted average of all future expected inflation and output variability is taken into account. With our approach policy makers are more "simple minded" as they care only about conditional medium term inflation and GDP growth volatility.

Another modeling issue is raised by the Lucas critique. Of course, our approach would be invalid if we considered changes in $\alpha$ as regime changes. We argue in a similar way against this possible objection as we did before when we briefly addressed the question of using data from a period of monetary targeting to estimate a model used for the analysis of 
forecast oriented monetary policy. We see "regime changes" as fundamental new orientations of the goals of monetary policy as the transition from an environment of high and volatile inflation to a policy of keeping inflation low as many countries experienced in the last 15 years. In our model inflation always returns to its long-run equilibrium irrespective of the value adopted for $\alpha$. We consider such changes therefore as "modest" and non-fundamental as the low inflation environment prevailing in Switzerland since the mid-seventies is preserved.

Now let us see to what extent the planned sequence of monetary policy shocks is able to close the deviation of average inflation and output growth rate from their targets. The remaining gaps $r p_{t+K}$ and $r y_{t+K}$ (measured as deviation from target) corresponds to the impact of the policy shocks minus the forecasted deviation from target $d_{p}(K, t)$ and $d_{y}(K, t)$ induced by the three non-policy shocks at time $t$. The cumulated impulse response function leads to the following deviations for inflation ( $r p$ ) and GDP growth (ry) from target, respectively (Kugler, Jordan, Lenz and Savioz, 2005):

$$
\begin{aligned}
& r p_{t+K}=\sum_{i=1}^{K} A A_{44}(K-i) u_{4 t+i}-d_{p}(K, t) \\
& r p_{t+K}=\sum_{i=1}^{K}(1-\alpha) \frac{A A_{14}(K-i) A A_{44}(K-i)}{\sum_{j=0}^{K-1} A A_{14}^{2}(j)} d_{y}(K, t)+\alpha d_{p}(K, t)-d_{p}(K, t) \\
& r p_{t+K}=(1-\alpha) G_{y} d_{y}(K, t)-(1-\alpha) d_{p}(K, t)=-(1-\alpha)\left[d_{p}(K, t)-G_{y} d_{y}(K, t)\right] \\
& r y_{t+K}=\sum_{i=1}^{K} A A_{14}(K-i) u_{4 T+i}-d_{y}(K, t) \\
& r y_{t+K}=\sum_{i=1}^{K} \alpha \frac{A A_{14}(K-i) A A_{44}(K-i)}{\sum_{j=0}^{K-1} A A_{44}^{2}(j)} d_{p}(K, t)+(1-\alpha) d_{y}(K, t)-d_{y}(K, t) \\
& r y_{t+K}=\alpha G_{p} d_{p}(K, t)+(1-\alpha) d_{y}(K, t)-d_{y}(K, t)=-\alpha\left[d_{y}(K, t)-G_{p} d_{p}(K, t)\right]
\end{aligned}
$$

For the economic interpretation of the two expressions derived above, we briefly consider for example the (expected) response of the medium term inflation rate to the forecast-oriented monetary policy. If $\alpha$ is equal to one (strict medium-term inflation targeting), we expect to hit the average inflation target exactly and the gap $r p_{t+K}$ is zero. Otherwise the medium term deviation of inflation from target is determined by the expression in brackets $\left[d_{p}(K, t)-G_{y} d_{y}(K, t)\right]$. The second term of this expression reflects the influence of the reaction of monetary policy to the output growth target, which depends on the deviation 
from the output target $d_{y}$ and the parameter $G_{y}$ which measures the co-movement of output and prices in reaction to a monetary policy shock.

The deviation of the $K$-period ahead average inflation and output growth from target is revised in period $t$ according to the shocks hitting the economy. For the sake of simplicity, we assume that we are in equilibrium in time $t-1$ in the sense that we expect to hit both targets in the period $t$ to $t+K-1$ and correspondingly the monetary policy shock in period $t$ is zero. However, the non-policy shock of period $t$ leads to deviations from the targets, which in turn needs a revision of the planned monetary policy shock sequence. We first note that the deviations of the unconditional forecasts from their targets are given by:

$$
\begin{aligned}
& d_{p}(K, t)=-\sum_{l \neq 4}\left(A A_{4 l}(K)-A A_{4 l}(0)\right) u_{l t} \\
& d_{y}(K, t)=-\sum_{l \neq 4}\left(A A_{1 l}(K)-A A_{1 l}(0)\right) u_{l t}
\end{aligned}
$$

Note that the structural shocks have an impact effect on inflation and output growth which has to be subtracted as it has no influence on future inflation and growth. Substituting these expressions into equation (6) and (7), respectively we get

$$
\begin{aligned}
& r p_{t+K}=(1-\alpha) \sum_{l \neq 4}\left[A A_{4 l}(K)-A A_{4 l}(0)-G_{y}\left(A A_{1 l}(K)-A A_{l l}(0)\right)\right] u_{l t} \\
& r y_{t+K}=\alpha \sum_{l \neq 4}\left[A A_{1 l}(K)-A A_{1 l}(0)-G_{p}\left(A A_{4 l}(K)-A A_{4 l}(0)\right)\right] u_{l t}
\end{aligned}
$$

Calculating conditional variances (given information of time $t$ ) provides the following expressions:

$$
\begin{aligned}
& \operatorname{Var}_{t}\left(r p_{t+K}\right)=(1-\alpha)^{2} \sum_{l \neq 4}\left[A A_{4 l}(K)-A A_{4 l}(0)-G_{y}\left(A A_{1 l}(K)-A A_{1 l}(0)\right)\right]^{2} \\
& \operatorname{Var}_{t}\left(r y_{t+K}\right)=\alpha^{2} \sum_{l \neq 4}\left[A A_{1 l}(K)-A A_{1 l}(0)-G_{p}\left(A A_{4 l}(K)-A A_{4 l}(0)\right)\right]^{2}
\end{aligned}
$$

This implies a linear trade-off in the standard deviations of the output growth and inflation medium-term responses. Of course, this conditional variance is zero for inflation (output growth) when $\alpha$ is $1(0)$, otherwise both variances are larger than zero. The reader has to be reminded that these conditional variances are with respect to the $K$-period ahead expected values in $t$. 


\section{The Effect of GDP Revision Errors on Inflation and Growth Rate}

\section{Volatility}

In this section we analyze the effects of GDP revision errors on monetary policy induced volatility of inflation and growth. For CPI inflation, data revision errors are not a serious problem given the fact that these data are available on a monthly basis practically without delay and are hardly ever revised. The same applies to money stock data which are only subject to minor revisions. However, data revision errors are clearly a problem for output: Two months after the end of a quarter a first GDP estimate is released. In September of year $t$ these figures for the preceding year are revised when they are adjusted to the first release annual GDP data for the preceding year. The annual account of year t-1 is itself subject to revisions which are published in September of year $\mathrm{t}+1$ and which lead to a second revision of quarterly GDP figures of year $\mathrm{t}-1$. Moreover, the changes in the base year of the account in 1989 and 1997 lead to additional differences between the final series available today and the real time data of before $1996^{4}$. This timing of data revisions implies that real time forecast in our VAR with lag length five have to be based on preliminary GPD figures which are all subject to revisions.

The first release quarterly GDP figures are based on a regression of annual GDP on annual data of quarterly available production indicators taking into account first order autoregression of the residuals. After annual data became available the difference between the annual figure and the sum of the quarterly first release figures is distributed to the quarterly data taking into account the autocorrelation of the residuals of the regression equation (Chow and Lin, 1971).

Swiss Quarterly GDP data are released regularly with a two months delay since 1980 when for the first time an official historical quarterly account going back to the mid-sixties was published. The difference (expressed in percentage points) between the final 1990 base year figure and the first release is plotted in Figure 2. The GDP data revision error appears as a highly volatile and strongly positively autocorrelated but stationary time series with a mean close to zero. Indeed a standard unit root test clearly rejects the null hypothesis and an AR(1) model with a coefficient close to 0.8 fits the data well. The estimates of the mean, which is 
not significantly different from zero, and the standard deviation are -0.13 and 1.16, respectively. The standard deviation for the data revision error of the growth rate of GDP, which is relevant in our VAR in first differences, is 0.81 . Moreover, there is some slight negative autocorrelation which is not statistically significant and which can be neglected for practical purposes. ${ }^{5}$ Before turning to the effects of these data revision errors on the volatility trade-off between inflation and GDP growth, we have to briefly discuss the high persistence of the data revision errors documented above. At first sight this seems to indicate that the first releases of the GDP figures are sub-optimal estimates. However, such a conclusion is not warranted for the following reason: the revision of the quarterly GDP figures within the framework of the Chow and Lin method leads by construction to autocorrelated revision errors. Moreover, a change in the base year when new annual figures for many previous years become available leads to persistent revision errors.

This GDP revision error has an influence on growth and inflation forecasts, which depend on $n$ (equal to the VAR lag length minus one) lagged noisy growth rates in our VAR framework. The easiest way to calculate the effect of the data revision errors on these forecasts is based on the reduced form vector moving average representation of the time $t+i$ $(\mathrm{i}=1,2 \ldots, \mathrm{K})$ value of the vector $X$ defined in section 2 :

$$
X_{t+i}=e_{t+i}+C(1) e_{t+i-1}+\ldots . .+C(i) e_{t}+C(i-1) e_{t-1}+\ldots+C(i-n) e_{t-n}+\ldots . .
$$

The data revision error in GDP growth (the first element of the vector $X$ ) in time $t$, say $v_{t}$, can be interpreted as a change in the first element of reduced form error $e$ in time $t$ and has, therefore, the effect $C_{11}(i)$ on the GDP growth forecast made in $t$, where $C_{11}(i)$ is the element 1,1 of the reduced form impulse response matrix $C(i)$. Similarly the effect on the inflation forecast is $C_{41}(i)$. If the data revision error is dated time $t-j$ the corresponding effects are $C_{l 1}(i-j),(l=1,4)$. In our framework we are interested in the cumulated effect of the data revision errors on the inflation and output growth forecast up to $K$ periods, which are given by

\footnotetext{
${ }^{4}$ These base year changes include conceptual changes in the annual account, in particular in 1996. However, we think that these changes also have to be accounted for as they should result in better GDP data which were calculated back to 1980 and which were used for the estimation of the model.

5 The first order autocorrelation coefficient is -0.3 .
} 


$$
\begin{aligned}
& d e_{p}(K, t)=\sum_{j=0}^{n} \sum_{i=1}^{K} C_{14}(i-j) v_{t-j} \\
& d e_{y}(K, t)=\left(\sum_{i=1}^{K} C_{11}(i)-1\right) v_{t}+\sum_{j=1}^{n} \sum_{i=1}^{K} C_{11}(i-j) v_{t-j}
\end{aligned}
$$

The current data revision error has a special effect on the forecasted target deviation of output as it effects the period $t$ as well as the expected period $t+K$ value log output in equation (3). This explains the subtraction of 1 in the first term of the second equation given above.

Let us now consider the data revision induced expected deviations from the inflation and growth target rep and rey, respectively. Monetary policy reacts to these deviations according to equation (5) given in Section 2, where $d$-terms are replaced by de-terms given in equations (15) and (16):

$$
u_{4 t+i}=\alpha g_{p i} d e_{p}(K, t)+(1-\alpha) g_{y i} d e_{y}(K, t), \quad i=1, \ldots, K
$$

Therefore, the expected deviation from target of inflation and growth after the (error) induced policy reaction are given by

$$
\begin{aligned}
& \operatorname{rep}_{t+K}=\sum_{i=1}^{K} A A_{44}(K-i) u_{4 t+i} \\
& \operatorname{rey}_{t+K}=\sum_{i=1}^{K} A A_{14}(K-i) u_{4 t+i}
\end{aligned}
$$

The evaluation of these expressions - analogous to the derivation of equations (6) and (7) in Section 2 - leads to the following two equations:

$$
\begin{aligned}
& \operatorname{rep}_{t+K}=\alpha d e_{p}(K, t)+(1-\alpha) G_{y} d e_{y}(K, t) \\
& \operatorname{rey}_{t+K}=\alpha G_{p} d e_{p}(K, t)+(1-\alpha) d e_{y}(K, t)
\end{aligned}
$$

Accordingly, we get the following expressions for the conditional (given time $t$ information) variances for these data revision error induced changes: 


$$
\begin{aligned}
& \operatorname{Var}\left(\operatorname{rep}_{t+K}\right)= \\
& \alpha^{2} \operatorname{Var}\left(d e_{p}(K, t)\right)+(1-\alpha)^{2} G_{y}{ }^{2} \operatorname{Var}\left(d e_{y}(K, t)\right)+2 \alpha(1-\alpha) G_{y} \operatorname{Cov}\left(d e_{p}(K, t), d e_{y}(K, t)\right) \\
& \operatorname{Var}\left(\operatorname{rey}_{t+K}\right)= \\
& \alpha^{2} G_{p}{ }^{2} \operatorname{Var}\left(d e_{p}(K, t)\right)+(1-\alpha)^{2} \operatorname{Var}\left(d e_{y}(K, t)\right)+2 \alpha(1-\alpha) G_{p} \operatorname{Cov}\left(d e_{p}(K, t), d e_{y}(K, t)\right)
\end{aligned}
$$

The covariance matrix of the data revision error induced deviations from target is easily obtained as follows: These two variables can be written as a linear transformation of the $n$ relevant error terms collected in a Vector $V$, namely $D V$. The elements of the $2 \mathrm{x} n$ matrix $D$ are obtained by the reduced form impulse responses as given above. Thus, the covariance matrix of the two deviations is $D \operatorname{Cov}(V) D^{\prime}$ where the covariance matrix of $V[\operatorname{Cov}(V)]$ is approximately diagonal, given the approximate white noise property of the data revision error. Therefore, the (reduced form) impulse response estimate of our SVAR model and the variance for the data revision error allow the calculation of the variances and the covariance of the two target deviations.

In Figures 3 and 4 the inflation and the output growth variance caused by the data revision error and the induced policy reaction is plotted as function of $\alpha$ for $K=8$ (which is favorable to output growth targeting as the effect of monetary policy on output at this horizon is relatively strong) and $n=4$, which is the relevant number of lagged data revision errors in our VAR model with lag length 5 . We can see that the minimum variance in both cases is now obtained with a value of $\alpha$ approximately equal to 0.75 . The declining segments of these graphs are brought about by the fact that the reduced form impulse response of inflation to a reduced form shock in output growth is relatively weak compared to that of output growth itself. The slight increase of the data revision error induced variability is caused by the negative correlation between responses of cumulated growth and inflation. The loss of this "diversification" effect leads to an increase in both variances when the weight of the strict medium-term inflation target gets extreme. However, note that the existence of data revision errors in GDP growth favor a higher weight for the strict medium-term inflation target.

Figure 5 includes 2 scatterplots for different values of $\alpha$ adopting a 8 quarters horizon $K$. The first refers to the conditional variance of output growth and inflation due to the occurrence of structural shocks as described in section 2 (equations 12 and 13). However, the findings are robust with respect to reasonable changes of the target horizon for the conduct of monetary policy (two to three and a half years). We can see from Figure 5 that our SVAR model implies a standard convex efficiency frontier for the conditional variances of inflation 
and growth. Tolerating a higher variability of output growth allows for a lower variability of inflation and vice versa (trade-off). Thereby, the maximum variability of inflation is clearly higher than the maximum variability of output growth. This result is caused by the higher persistence of the impact of the shocks on inflation than on growth. The second scatterplot adds the variance due to data revision errors. We note that there is no longer a convex efficiency frontier if data revision errors in GDP are taken into account: Decreasing the weight of output growth targeting over some range, i.e., increasing $\alpha$ from 0 to approximately 0.42 , decreases the conditional variance of both inflation and growth. Thus, there is no trade-off between smaller output growth variance and higher inflation variance over this range. This result is due to the fact that with data revision errors, monetary policy reacts too much to noisy data if the weight on output growth targeting becomes too big. This occurs because the data revision error has a strong impact on the growth forecast but not on the inflation forecast. However, if $\alpha>0.85$ this effect is slightly out-weighted by a loss of "diversification" which is brought about by the negative correlation of the data revision error induced responses of medium term inflation and growth.

The non-convex efficiency frontier is an empirical result, but the same phenomenon may arise in a standard New-Keynesian model of Clarida, Gali and Gertler (1999) when the central bank reacts to noisy output gap data. The corresponding analysis is briefly presented in an appendix to this paper. Of course this raises the question why the central bank does not account for the errors in the GDP data in its policy by constructing an optimal estimate of GDP given all available information. Our approach can be defended against this objection by two arguments: first, the quarterly GDP figures released by the statistical office should be a "best" estimate using all quarterly available production, employment and survey information. Thus, it is not easy to argue that the central bank is able to provide better quarterly GDP estimates. Second, even if the central bank could produce better GDP estimates it would be very difficult to communicate monetary policy decision relying on GDP figures which are different from the official data provided by the government's statistical office. Such an approach would obviously lead to credibility problems for the central bank. Note that for the output gap this problem does not arise, as there is no official figures released for this variable and the central bank has the freedom to produce its own estimate. In sum we believe that the central bank has to base its policy on the officially released GDP figures when it adopts a GDP growth target. 


\section{Non-Equilibrium Initial Conditions and Policy Dynamics}

So far we have focussed our analysis on the effects of time $t$ structural shocks on the time $t$ expected (conditional) volatility of the $K$-period ahead expected inflation and growth rate given long-run equilibrium in the past in the sense that we assume that all shocks dated earlier than $t$ are zero. The results obtained under this assumption may differ from those for the unconditional variance of medium-term inflation and growth for two reasons: first, there is no long-run equilibrium in the past and past shocks influence current and future outcomes. Second the realized period $t+K$ outcome depends, of course, also on the shocks and policy decisions in the period $t+1, t+2, \ldots, t+K$ which depend on the future medium-term inflation and growth forecasts. Moreover, future policy decisions are influenced by current policy decisions which do not only have an effect on average inflation and growth up to $t+K$ but also affect time $t+K+1, t+K+2, \ldots$, outcomes.

In order to investigate the effects of different monetary policy strategies on the unconditional variance of medium-term inflation and growth, we use a simulation approach. We take the history of the three structural shocks from 1982 to 2002 and the initial conditions in 1980/81 as given and simulate the development of the four variables of our SVAR model under the assumption that the monetary policy shocks are generated according to Equation (5) for different values of $\alpha$ and using historical averages as inflation and growth targets ${ }^{6}$. The counterfactual series obtained in this way are then used to calculate the variance of mediumterm inflation and growth (the sample variance of the generated series $x_{t+K}^{g}-x_{t}^{g}$ ) for varying values of $\alpha$. The generation of the forecasts guiding monetary policy is done firstly by neglecting the GDP revision errors and secondly by using the real time data. The scatter diagrams obtained from these simulations are displayed in Figure 6.

Figure 6 differs from Figure 5 with two respects: first, the unconditional variance is substantially (nearly ten times) higher than the time $t$ variance. Second, the efficiency frontier is no longer convex but slightly concave in the unconditional case without data revision errors. This result is brought about by a differing shape of the dependence of the inflation and growth variance on $\alpha$. The variance of inflation (growth) decreases (increases) with $\alpha$ at a decreasing rate. Therefore, lowering the ouput (inflation) weight in a situation of strict medium-term growth (inflation) targeting leads to a strong (weak) reduction of growth 
(inflation) variance. The first difference is easy to explain since not only current (time $t$ ) shocks are taken into account but also past and future (up to time $t+K$ ) shocks are accounted for. The non-convex shape of the is basically explained by the fact that in our framework monetary policy aims at a low ex ante variability of the time $t$ expected medium term growth and inflation rate and not at the ex post variability of these series. The unconditional variance depends in a much more complex way on $\alpha$ than the variance of the time $t$ expected variances given in Section 2. In order to see this complexity it is helpful to consider first the unconditional (no initial equilibrium) deviations from targets which are given by the following equations:

$$
\begin{aligned}
& \left.d_{p}(K, t)=-\sum_{j=0}^{\infty}\left\{\left(A A_{4 l}(K+j)-A A_{4 l}(j)\right) u_{4 t-j}+\sum_{l=1}^{3}\left(A A_{4 l}(K+j)-A A_{4 l}(j)\right) u_{l t-j}\right)\right\} \\
& \left.d_{y}(K, t)=-\sum_{j=0}^{\infty}\left\{\left(A A_{1 l}(K+j)-A A_{1 l}(j)\right) u_{4 t-j}+\sum_{l=1}^{3}\left(A A_{1 l}(K+j)-A A_{1 l}(j)\right) u_{l t-j}\right)\right\}
\end{aligned}
$$

The first term in the two equations above gives the effect of past structural and policy shocks on the forecast deviations from target in $t+1$. Under the initial equilibrium assumption these expressions reduce to (8) and (9) as only the time $t$ structural shocks are different from zero. When these equations are combined with (5) for $i$ equal to 1 , $u_{4 t+1}=\alpha g_{p 1} d_{p}(K, t)+(1-\alpha) g_{y 1} d_{y}(K, t)$, we see that we generate an autoregressive dependence of the policy shock:

$$
\begin{aligned}
u_{4 t+1}= & -\sum_{j=1}^{\infty}\left\{\left(\alpha g_{p 1}\left(A A_{4 l}(K+j)-A A_{4 l}(j)\right)+(1-\alpha) g_{y 1}\left(A A_{1 l}(K+j)-A A_{1 l}(j)\right)\right) u_{4 t-j}+\right. \\
& \left.\left.\sum_{l=1}^{3} \alpha g_{p 1}\left(A A_{4 l}(K+j)-A A_{4 l}(j)\right)+(1-\alpha) g_{y 1}\left(A A_{1 l}(K+j)-A A_{1 l}(j)\right)\right) u_{l t-j}\right\}
\end{aligned}
$$

The AR coefficients depend on the $K$ period differences of the cumulated impulse responses of inflation and growth, the weight of strict medium-term inflation targeting and the t+1 policy response under strict medium-term inflation and growth targeting. As the difference of the cumulated inflation impulse response is always positive, we have a negative AR dependence (positive) in the case of strict medium-term inflation (growth) targeting ${ }^{7}$. To

\footnotetext{
${ }^{6}$ The values are 2.4 and 1.4 percent per annum for inflation and growth, respectively.

${ }^{7}$ Note that the differing sizes of the cumulated impulse responses of inflation and growth are approximately compensated by different value of the g-coefficient which is close to $1 / 3$ and $3 / 4$ for inflation and growth, respectively.
} 
understand this result intuitively, consider a currently expansive monetary policy which leads to an increase in output which is reversed after seven quarters. The reversion of the policy effect calls for an expansionary policy in the future in order to hit the growth target. Therefore, strict medium-term growth targeting can generate self-enforcing policy actions. The same story applies to policy actions to current IS and money demand shocks which have the same hump-shaped cumulated response pattern of GDP growth. This effect plays no role for strict medium-term inflation targeting, as we have a permanent effect of all shocks on the price level which is reached smoothly over time.

The arguments outlined above indicate that there is a difference between the trade-off in the dynamic context considered in this section and the trade-off in the "static" context of Section 2. In the fully dynamic context, large (small) reductions in inflation variability always have to be paid more or less by large (small) increases in GDP growth variability. The second scatter plot of Figure 6 shows that the dynamic effects of the GDP-data revision error lead to an even more pronounced non-standard efficiency frontier. It has an increasing branch for $\alpha$ between 0.45 and 1 . This result is brought about by the strong influence of the data revision errors on the GDP forecasts and the implied policy reaction which creates higher inflation and GDP growth variability. This effect out-weighs the small reduction in growth rate variability caused by the decrease of $\alpha$. For values of $\alpha$ smaller than 0.45 , the impact of a lower $\alpha$ dominates the impact of data revision errors and thus leads to a strong reduction of growth variability. However, most of this branch is not feasible as the assumption of moderate policy intervention is clearly no longer appropriate. These outcomes require monetary policy shocks that are clearly positive on average and thus invalidate our analysis. In other words, with values of $\alpha$ smaller than 0.3 , simulated average inflation rate is between 0.5 to 1.5 percent points per annum above its historical value ${ }^{8}$. Therefore, the consideration of GDP data revision errors in our fully dynamic context sharpens our policy conclusion of Section 3 strongly in the sense that strict-medium term inflation targeting is suggested as a strategy to get inflation and growth variability as low as possible.

\footnotetext{
${ }^{8}$ It should be stressed that for all other simulations we get average monetary policy shocks which are essentially 0 and therefore average simulated inflation rates which are very close to the historical average.
} 


\section{Conclusions}

In this paper we analyzed forward-looking rules for Swiss monetary policy in a small structural VAR consisting of four variables in the presence of GDP revision errors. There are two main results of the paper. First, if data revision errors in GDP are taken into account, there is no longer a convex efficiency frontier between the conditional variance of mediumterm inflation and GDP growth: Increasing the weight of the output growth target $(1-\alpha)$ from 0.58 to 1 increases the conditional variance of both medium-term expected inflation and growth. This result is due to the fact that with, data revision errors, monetary policy reacts too strongly to noisy data if the weight on output growth targeting becomes too big, as data revision errors have a strong impact on the growth forecast but not on the inflation forecast. However, if $\alpha>0.82$, this effect is slightly out-weighted by a loss of "diversification" which is brought about by the negative correlation of the policy reaction to data revision error induced responses of medium-term inflation and growth. Therefore, a strict medium-term inflation strategy is inefficient even if the costs of increased volatility of inflation and growth compared to the case $\alpha=0.82$ are relatively small.

The second result shows that this effect of data revision error is reinforced when nonequilibrium initial conditions and the consequences of endogenous policy dynamics are taken into account. In fact, in the presence of GDP data revision errors, policy reactions to the inflation and growth consequences of past policy decisions may even destabilize the economy if the weight on the medium-term GDP growth target is high. In general, the paper indicates that under realistic assumptions the central bank induces a higher variability of both output growth and inflation by concentrating too strongly on output growth. The existence of data revision errors for GDP forcefully underline the limits and the risks of a monetary policy aiming at output stabilization. Even if the central bank only cares about growth stabilization, the weight on this target relative to the one of the inflation target should be clearly smaller than 1 or even zero. Thus, our results confirm the concerns about output growth stabilization first raised by Orphanides (2000) when real time data problems and are taken into account. In addition our findings are in line with the theoretical analysis of Gaspar and Vestin (2004) who consider a non-optimizing policy maker following reasonable policy precepts in a standard new Keynesian model with uncertainty about the output gap.

The results of the paper can also be linked to the literature on time-inconsistency of monetary policy of the type introduced by Barro and Gordon (1983) and Kydland and 
Prescott (1977). ${ }^{9}$ In a seminal paper Rogoff (1985) showed in this context that delegating monetary policy to a conservative central banker, i.e. a central banker who is relatively more concerned about inflation than the society as a whole, can decrease the variance of both inflation and output growth and thereby improve the welfare of the society. In our model, time-inconsistency is not a problem. However, we can interpret $\alpha$ as the fraction of inflation hawks in a central bank board. Thus, even if the society had strong preference of output stabilization, i.e. if $\alpha$ is 0 , it would be an advantage for the society to appoint a conservative board with a $\alpha$ clearly bigger than 0 . Such a board may deliver a smaller variability of output growth than a board that reflects exactly the preferences of the public. Consequently, our results support the view that the government should appoint conservative central bankers when it is politically difficult to motivate a downgrading of the medium term GDP growth target by data revision problems for GDP.

\section{References}

Barro, R.J. \& Gordon D.B. (1983). Rules, Discretion and Reputation in a Model of Monetary Policy. Journal of Monetary Economics ,12, 101-121.

Chow, G.C.\& Lin A. (1971). Best Linear Unbiased Distribution and Interpolation of Time Series by Related Series. Review of Economics and Statistics , 53, 372-375.

Clarida, R., Gali, J. \& Gertler M. (1999). The Science of Monetary Policy: A New Keynesian Perspective. Journal of Economic Literature, 37, 1661-1707.

Ehrmann, M. \& Smets, F. (2003). Uncertain Potential Output: Implications for Monetary Policy. Journal of Economic Dynamics and Control, 27: 1611-1638.

Gaspar, V. \& Vestin, D. (2004). Imperfect Kowledge, Learning and Conservatism, mimeo, Frankfurt, January 2004.

Jordan, T.J., Kugler, P., Lenz, C. \& Savioz, M.R. (2002). Inflationsprognosen mit vektorautoregressiven Modellen. Quartalsheft der Schweizerischen Nationalbank, 20(1), 41-66.

Jordan, T.J. \& Peytrignet, M. (2001). Die Inflationsprognose der Schweizerischen Nationalbank. Quartalsheft der Schweizerischen Nationalbank, 20(2), 54-61.

Kugler, P. \& Jordan, T.J. (2004), Vector Autoregressions and the Analysis of Monetary Policy Interventions: The Swiss Case. Schweizerische Zeitschrift für Volkswirtschaft und Statistik, $140,67-87$.

Kugler, P., Jordan, T.J., Lenz, C. \& Savioz, M.R. (2005). The Analysis of Forward-Looking Monetary Policy in a VAR Framework, SNB Working Paper Series, May 2005.

\footnotetext{
${ }^{9}$ As mentioned in the introduction the same result was obtained by Ehrmann and Smets (2003) in a different modelling framework.
} 
Kugler, P. \& Rich, G. (2002). Monetary Policy under Low Interest Rates: The Experience of Switzerland in the late 1970`s, Schweizerische Zeitschrift für Volkswirtschaft und Statistik, 138, 241-269.

Kydland, F.E. \& Prescott, E.C. (1977). Rules Rather Than Discretion: The Inconsistency of Optimal Plans. Journal of Political Economy, 85, 273-286.

Leeper, E.M. \& Zha, T. (1999). Modest Policy Interventions. Federal Reserve Bank of Atlanta Working Paper 99-122.

Lucas, R.E. (1976). Macroeconomic Policy Evaluation: A Critique. Carnegie Rochester Conference Series on Public Policy, 1, 19-46.

Rogoff, K. (1985). The Optimal Degree of Commitment to an Intermediate Monetary Target.

Quarterly Journal of Economics, 100, 1169-1189.

Orphanides, A. (2000). The Quest for Prosperity without Inflation. ECB Working Paper No. 15.

Orphanides, A.. (2001). Monetary Policy Rules Based on Real-Time Data. American Economic Review, 91, 964-985.

Smets, F. (2002). Output Gap Uncertainty: does it Matter for the Taylor Rule. Empirical Economics, 27, 113-129.

Stalder, P. (2001). Ein ökonometrisches Makromodell für die Schweiz. Quartalsheft der Schweizerischen Nationalbank, 19(2):63-89.

Svenssson, L. \& Woodford, M. (2000). Indicator Variables for Optimal Monetary Policy. ECB Working Paper No. 12.

\section{Appendix: GDP Revision Errors in a Simple Theoretical Model}

Let us consider the standard New Keynesian model discussed in the survey paper of Clarida, Gali and Gertler (1999). It consists of IS-curve, a "new" Phillips curve and a quadratic loss function in inflation $\pi$ and growth or output gap $x$ of the central bank, which operates by setting the nominal interest rate $i$

$$
\begin{aligned}
& x_{t}=-\varphi\left(i_{t}-E_{t} \pi_{t+1}\right)+E_{t} x_{t+1}+g_{t}, \\
& \pi_{t}=\lambda x_{t}+\beta E_{t} \pi_{t+1}+u_{t} \\
& \frac{1}{2} E_{t} \sum_{i=0}^{\infty} \beta^{i}\left[a x_{t+i}^{2}+\pi_{t+i}^{2}\right]
\end{aligned},
$$

where $g$ and $u$ are zero mean structural shocks which follow an AR(1) process with coefficient $\mu$ and $\rho$, respectively.

In this model optimal monetary policy under discretion is characterized by the following first order condition

$$
x_{t}=-\frac{\lambda}{a} \pi_{t}
$$

implying that demand is contracted by increasing (decreasing) the interest rate when inflation is above (below) target which is supposed to be zero. Now let us assume that the central bank 
observes $x$ only with a zero mean data revision error $e$ which follows an AR(1) process with coefficient $\theta$. Therefore, the first order condition fulfilled by the central bank, which neglects this data revision problem, is

$$
x_{t}+e_{t}=-\frac{\lambda}{a} \pi_{t}
$$

Inserting the optimality condition in the Phillips curve results in

$$
\pi_{t}=-\lambda\left(\frac{\lambda}{a} \pi_{t}+e_{t}\right)+\beta E_{t} \pi_{t+1}+u_{t}
$$

The model solution is a linear function of the two state variables $u$ and $e$ with unknown coefficients:

$\pi_{t}=\phi_{1} u_{t}+\phi_{2} e_{t}$

According to the assumed $\mathrm{AR}(1)$ structure, the expected value for $\mathrm{t}+1$ is equal to

$$
E_{t} \pi_{t+1}=\phi_{1} \rho u_{t}+\phi_{2} \theta e_{t}
$$

Inserting these expressions in the first equation provides

$$
\left(1+\frac{\lambda^{2}}{a}\right)\left(\phi_{1} u_{t}+\phi_{2} e_{t}\right)=\beta\left(\phi_{1} \rho u_{t}+\phi_{2} \theta e_{t}\right)+u_{t}-\lambda e_{t}
$$

Equating coefficients on both sides of this equation results in

$$
\begin{aligned}
& \phi_{1}=\frac{a}{\lambda^{2}+a(1-\beta \rho)} \\
& \phi_{2}=-\frac{\lambda a}{\lambda^{2}+a(1-\beta \theta)}
\end{aligned}
$$

Inserting the solution for $\pi$ in the optimality condition easily results in the solution for $x$ and we get

$$
\begin{aligned}
& \pi_{t}=\frac{a}{\lambda^{2}+a(1-\beta \rho)} u_{t}-\frac{\lambda a}{\lambda^{2}+a(1-\beta \theta)} e_{t} \\
& x_{t}=-\frac{\lambda}{\lambda^{2}+a(1-\beta \rho)} u_{t}-\frac{a(1-\beta \theta)}{\lambda^{2}+a(1-\beta \theta)} e_{t}
\end{aligned}
$$

This means that a positive data revision error (growth is deemed to be higher by the central bank than it really is) leads to a too restrictive monetary policy resulting in lower growth and lower inflation. Under the (reasonable) assumption that $u$ and $e$ are not correlated we have the following expressions for the variances of inflation and growth

$$
\begin{aligned}
& \sigma_{\pi}^{2}=\left(\frac{a}{\lambda^{2}+a(1-\beta \rho)}\right)^{2} \sigma_{u}^{2}+\left(\frac{\lambda a}{\lambda^{2}+a(1-\beta \theta)}\right)^{2} \sigma_{e}^{2} \\
& \sigma_{x}^{2}=\left(\frac{\lambda}{\lambda^{2}+a(1-\beta \rho)}\right)^{2} \sigma_{u}^{2}+\left(\frac{a(1-\beta \theta)}{\lambda^{2}+a(1-\beta \theta)}\right)^{2} \sigma_{e}^{2}
\end{aligned}
$$


where $\sigma$ is the standard deviation of the variable indicated by the lower case letter. When there is no data revision error these expressions imply the standard convex policy efficiency frontier in the variance of growth and inflation which is obtained by varying $\alpha$ from zero to infinity as discussed by Clarida, Gali and Gertler. However, this convexity may be destroyed by the introduction of the GDP data revision error as monetary policy reacts on noisy output data. It can be easily seen from the above expressions that the contribution of the data revision error to the variance of inflation increases with $\alpha$ for both variables. Therefore, increasing a definitely increases the variance of inflation but the effect on the variance of growth is ambiguous: on the one hand the effect of the structural shock and the corresponding variability decreases. On the other hand the effect of the data revision error on output and correspondingly its variability increases. Which of these two effects dominates particularly depends on the relative magnitude of the variance of the structural shock and the data revision error.

Figure 1: Accumulated impulse responses of changes in log GDP, interest rate (3M-SFR-

\section{LIBOR), log real money stock (M1) and log prices (CPI) to structural shock, SVAR(5),}

quarterly data 1974/I-2002/IV
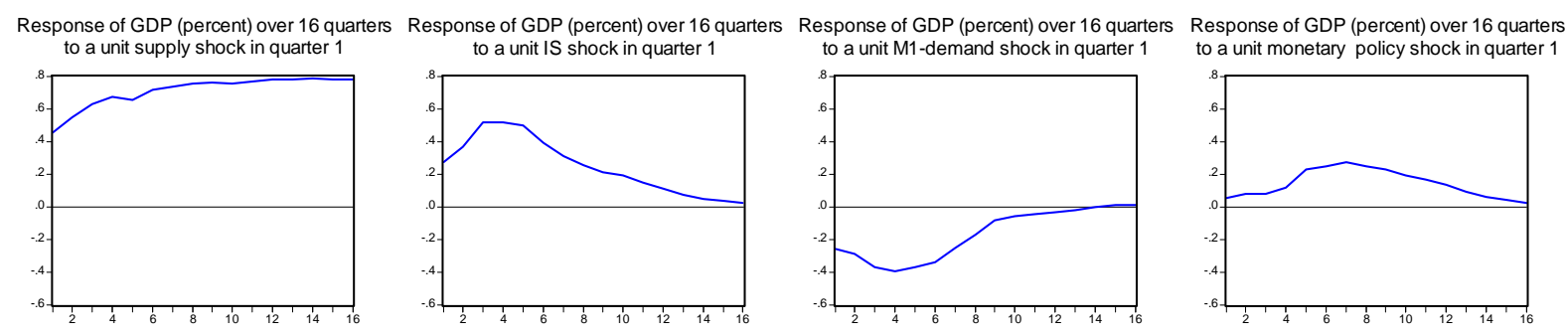

Response of LIBOR (percent) over 16 qua to a unit supply shock in quarter 1
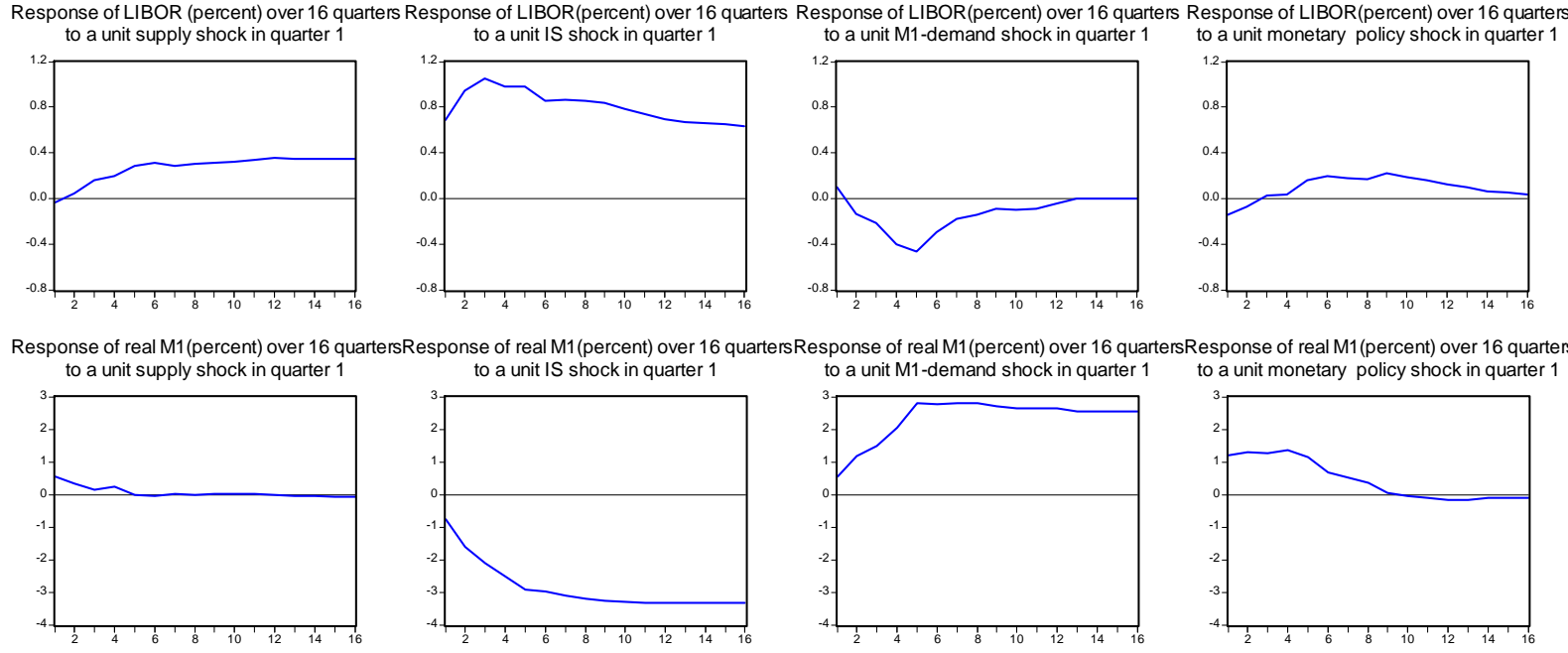

Response of CPI (percent) over 16 quarte
to a unit supply shock in quarter 1
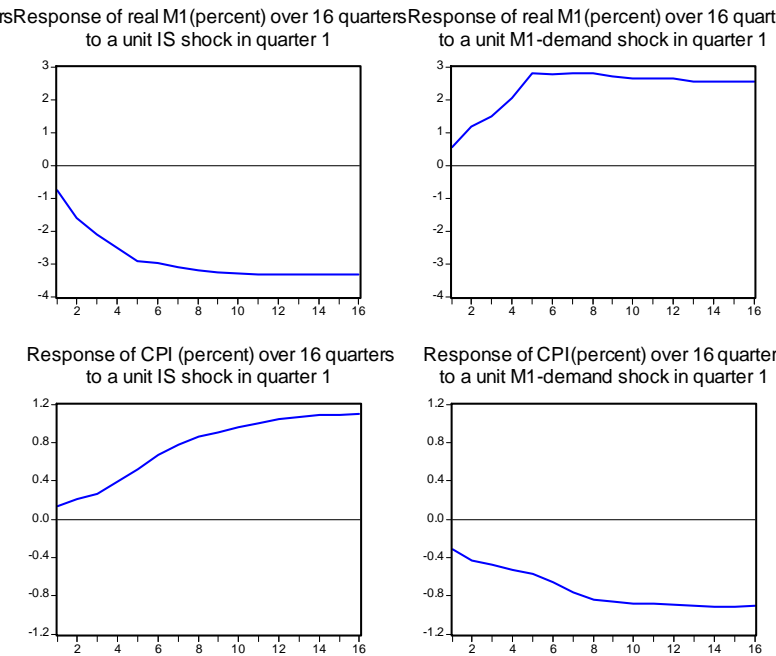

Response of real $\mathrm{M} 1$ (percent) over 16 quarters
to a unit monetary policy shock in quarter 1
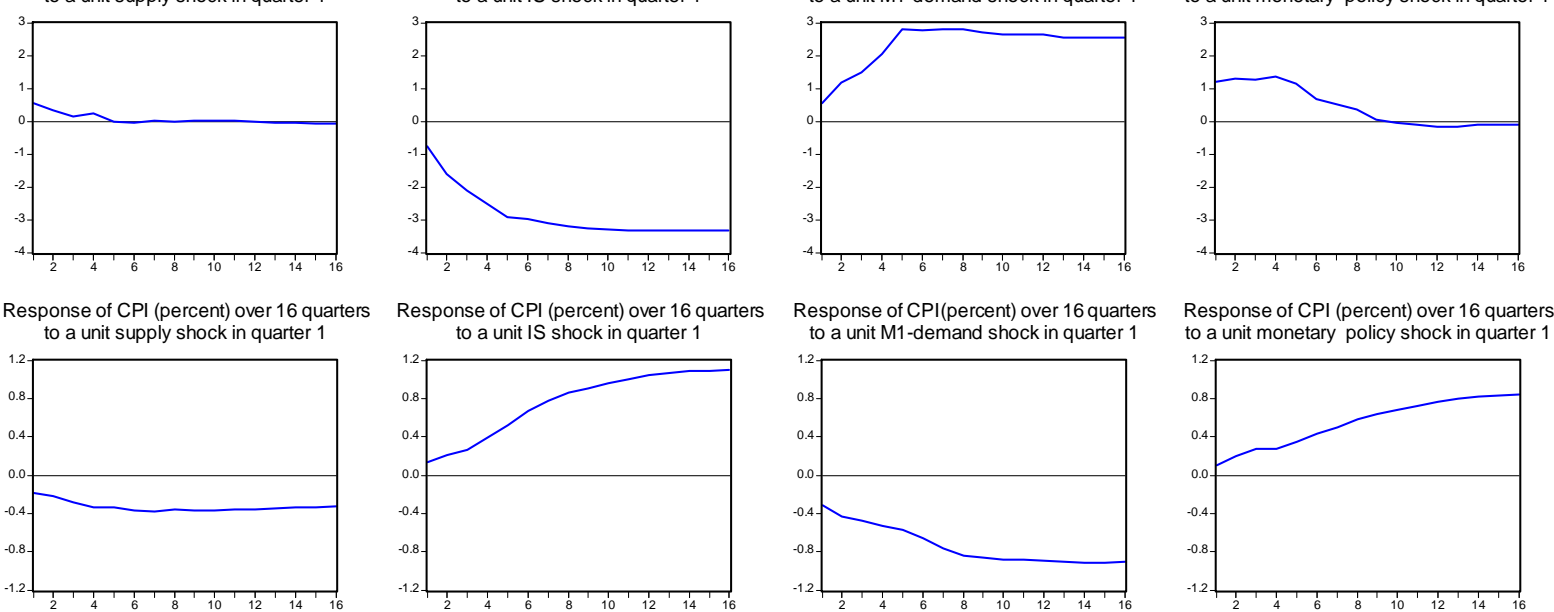

Response of CPI (percent) over 16 quarters to a unit monetary policy shock in quarter 1

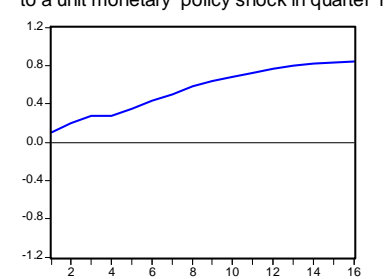


Figure 2: Data revision errors of quarterly Swiss GDP, 1980-2002

Percent difference between final GDP data and first released GDP data

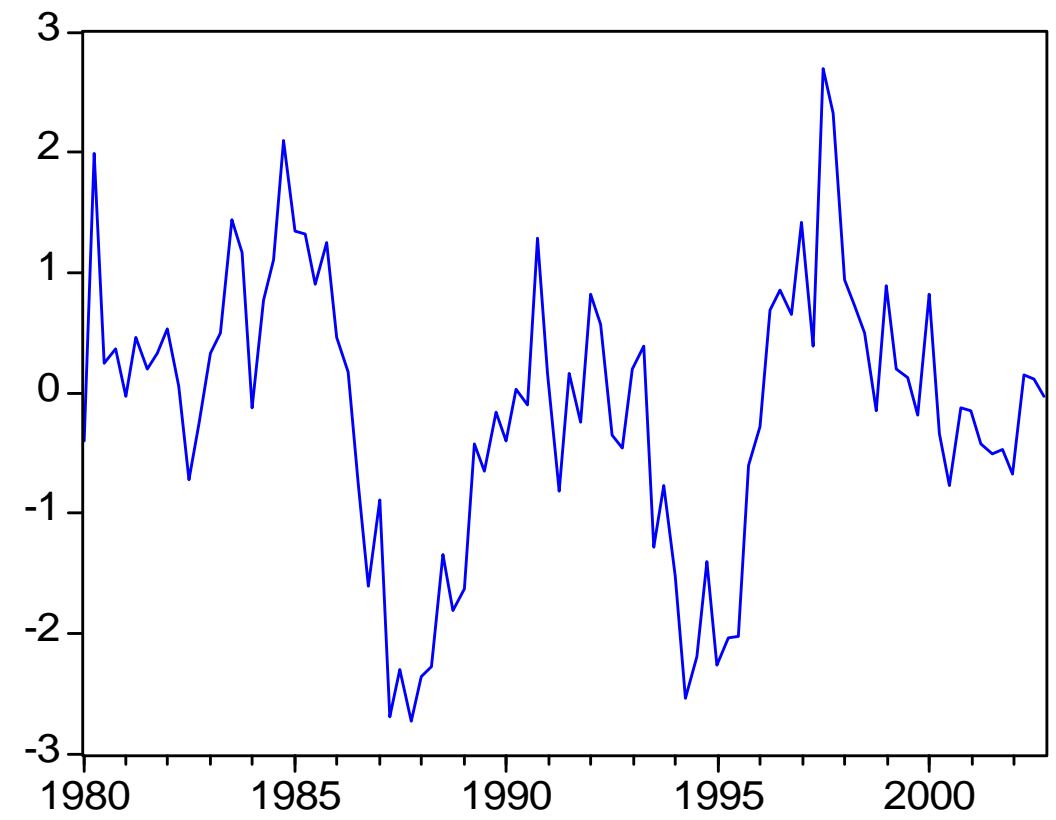

Figure 3: 8-quarters-ahead conditional variance of inflation caused by data revision errors of GDP

Inflation measured in percent, ALPHA is the weight of medium term inflation target

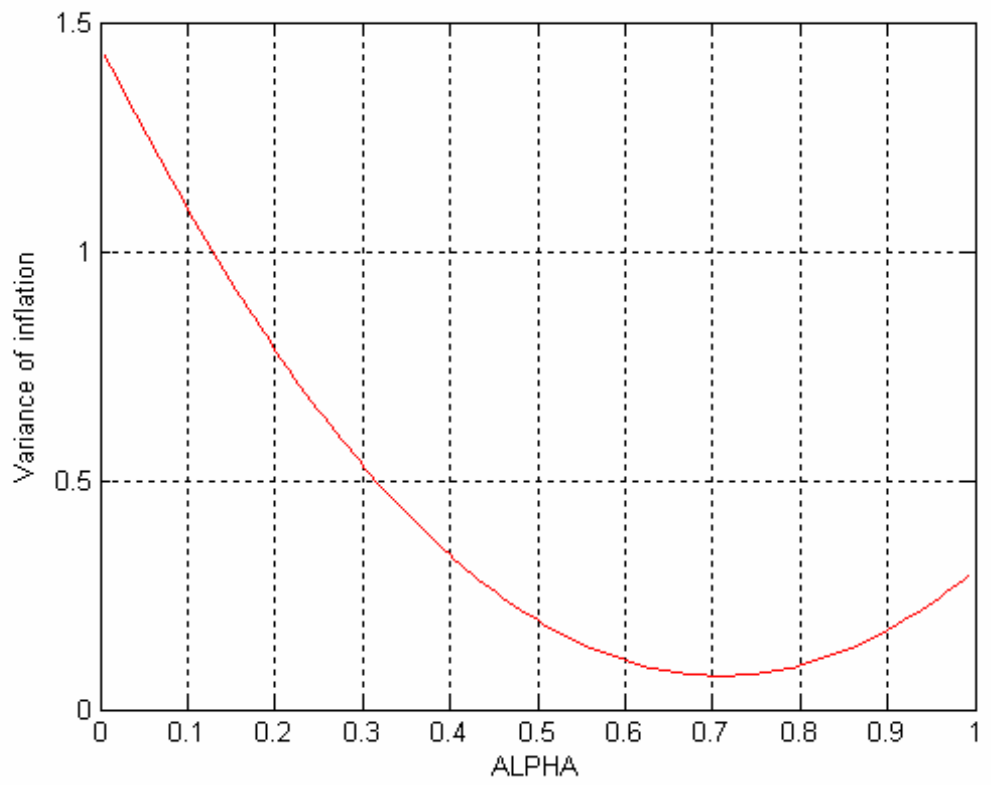


Figure 4: 8-quarters-ahead conditional variance of output growth caused by data revision errors of GDP

Output growth measured in percent, ALPHA is the weight of medium term inflation target

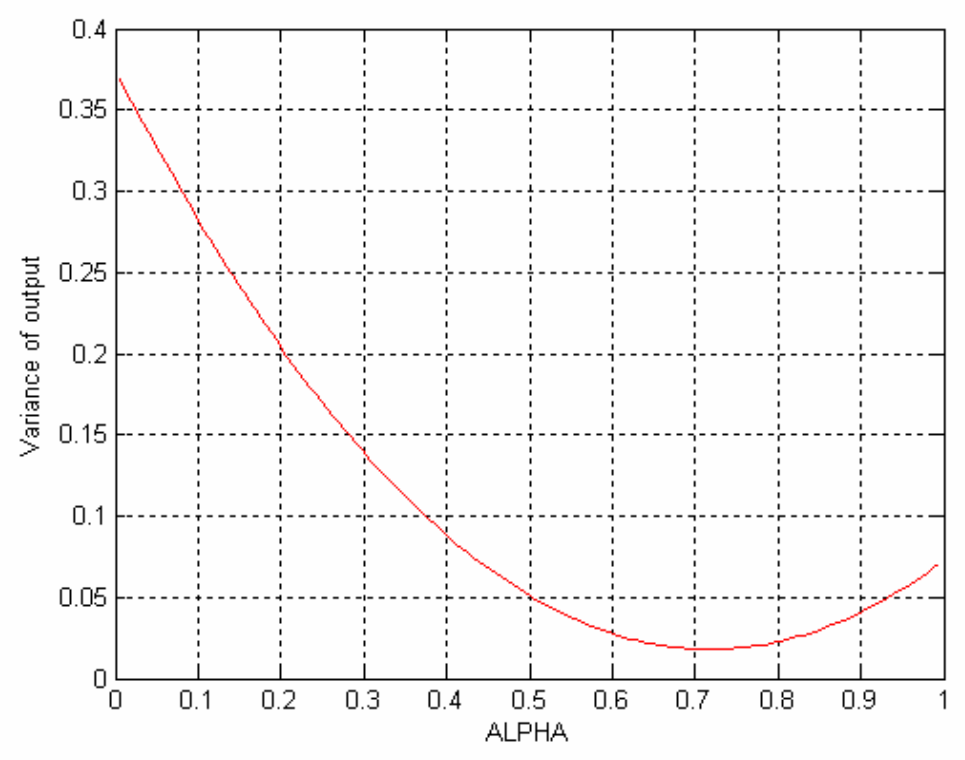

Figure 5: 8-quarters-ahead conditional variance of inflation and output growth trade-off

Structural shocks (dashed line) as well as structural shocks with varying $\alpha$ and data revision errors (solid line), inflation and output growth measured in percent; alpha is the weight of medium term inflation target

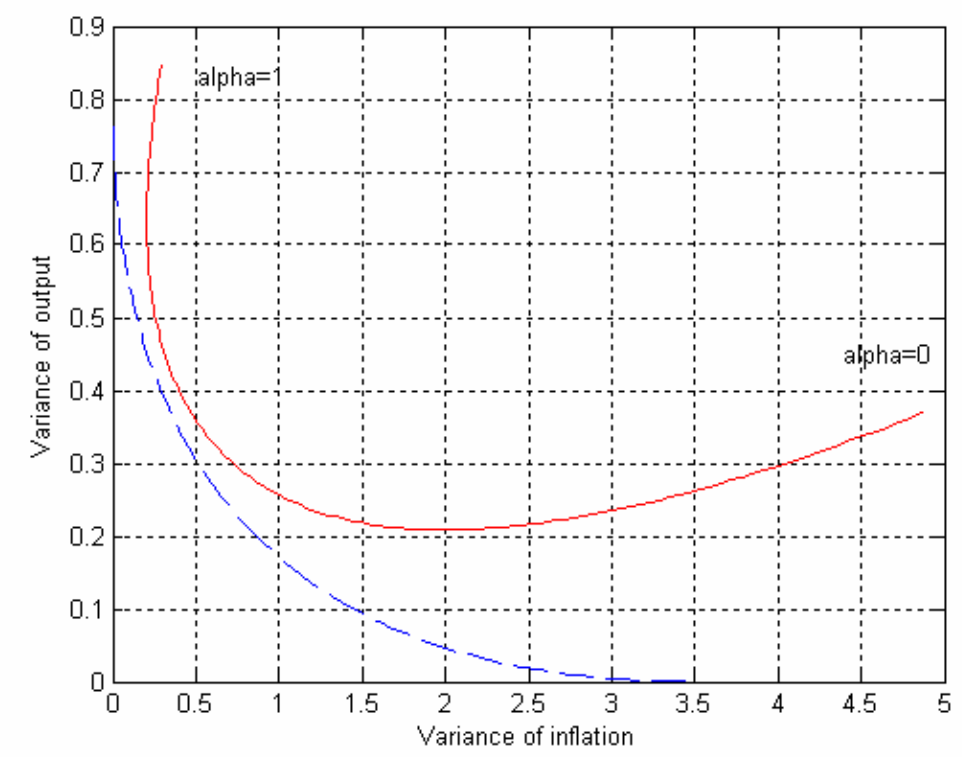


Figure 6: 8-quarters-ahead unconditional variance of inflation and output growth

\section{trade-off}

Historical structural shocks and simulated monetary policy with varying $\alpha$ (dashed line) as well as GDP data revision errors (solid line), inflation and output growth measured in percent; alpha is the weight of medium term inflation target

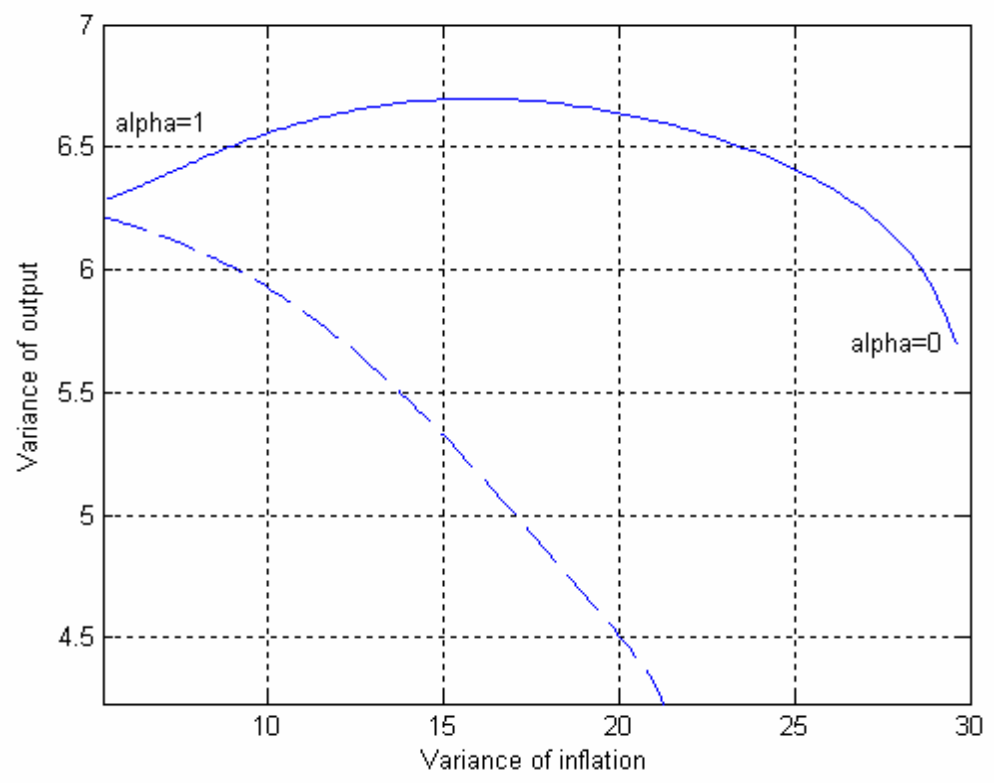

